\title{
On Achilles Heel of Some Optical Network Designs and Performance Comparisons
}

\author{
Dao Thanh Hai
}

Received: date / Accepted: date

\begin{abstract}
This non-conventional paper represents the first attempt to uncover a possible vulnerability in some proposals for optical network designs and performance comparisons. While optical network designs and planning lie at the heart of achieving fiber capacity efficiency and/or operational efficiency, its combinatorial nature makes it computationally hard to reach optimal solutions for realistic scenarios. As a consequence, the wellestablished way that have been taken for granted by not-so-small number of research papers is that an optimization model based on mixed integer linear programming (MILP) formulation is first proposed and then due to the intractability of such combinatorial optimization model, a heuristic algorithm is offered as an approximation. The solution-quality comparison between the MILP and heuristic is then carried out on small-scale instances including topologies and traffic tests to verify the efficacy of the proposed heuristic. Next such allegedly verified heuristic are used for optical network designs of realistic scenarios. This approach may nevertheless leave a critical vulnerability as there is no guarantee that one performs well in small tests will generalize adequately for large-scale cases, a common pitfall widely referred as the peril of extrapolation and/or overfitting. Besides, it is not uncommon that in some research works, for benchmarking purpose, the comparison between a new design proposal whose performance is obtained from on one heuristic and a reference design based on another heuristic is carried out. As the consequence of lacking solution quality check, such performance comparison relied merely on heuristic solutions may be equally vulnerable, resulting to possibly unre-
\end{abstract}

F. Author

Post and Telecommunication Institute of Technology

E-mail: haidt102@gmail.com liable conclusions. In this work, we pinpoint those issues and provide a realistic case study to highlight and demonstrate the impact of such vulnerabilities.

Keywords WDM networks · Elastic optical networks · routing and wavelength assignment · integer linear programming · heuristics · Achilles heel · performance evaluation

\section{Introduction}

Internet has become clearly the largest engineered system made by humankind with millions of end devices, telecommunication links, switches and routers connecting to each others and billions of users have been on Internet via different means. Less well-known is that the major segment of Internet infrastructure are composed of several billion kilometers of optical fibers that have been installed worldwide. Indeed, optical fibers serve as the tremendous communication channels through which nearly all voice, video, and data communications fly almost instantaneously around the globe [1,2]. For many years, the capacity of an optical fiber has been viewed as being almost infinite and indeed, the single channel capacity in optical core networks has undergone leap-and-bound growth with a spectacular rise from 2.5 $\mathrm{Gb} / \mathrm{s}$ in around 1990 to beyond $1 \mathrm{~Tb} / \mathrm{s}$ in 2020 [2,3]. Such factor of 400-fold increase in a span of roughly 30 years is thanks to convergence of remarkable advances in electronic, photonic and digital signal processing technologies [4 10]. Nevertheless, in order to exploit such huge capacity in an efficient and sustainable manner, great emphasis has been placed on developing efficient network design algorithms 11-18. Carefully designed optical networking algorithms have therefore 
emerged as one of the critical components accompanying system capacity growth. Clearly, it has been wellknown that a good set of algorithms will maximize the utilization of network resources, which ultimately translates to greater capital and operation efficiency. In contrast, inadequate algorithms may result in squandering the available capacity, causing poor network performances 19 24]

When it comes to designing and operating an optical network, a central issue to be addressed is the routing and resource allocations for demands 25 35. That problem will become basically the routing and spectrum assignment (RSA) if elastic optical networks technology is employed [36 40. In the case of operating with fixed wavelength division multiplexing (WDM) technology, it is called routing and wavelength assignment (RWA) problem [3,41. Although there have been various other variants depending on the physical layer assumptions and switching architectures of optical networks 5, 42,44, RWA and RSA remain the most basic issue on which other variants are emerged. It has to be noticed that the typical procedure that have been adopted in some research papers for dealing with RWA, RSA problems and other variants follow basically the same route as following:

- Step 1: Formulate the network design problem in the form of MILP model

- Step 2: Complete the formulation part with a conclusion whose meaning basically is the MILP model is not well-suited for a large number of traffic demands in large-scale network topologies and therefore heuristic algorithms are needed to develop

- Step 3: Develop one (or some) scalable heuristic algorithm

- Step 4: Test the efficacy of one (or some) proposed heurisic by comparing its solution with that of the MILP model in a small network topology and few demands; Declare the goodness of one proposed heurisic based on the data that its solution is close to or equal to optimal ones in small-scale tests and use that victory heuristic for designing and operating the realistic (large-scale) ones

- Step 5: Conduct a performance comparison on realistic network topologies between a new design proposal whose performance is obtained from the proposed heuristic and a reference design whose performance may be obtained from another heuristic in literature. The purpose is to report the achieved gains with new proposal compared to the reference one
That conventional process governing a number of research papers in optical network design and performance evaluation may have some vulnerabilities. First it remains uncertain that a heuristic that produces good results on a very small network would work adequately for a realistically sized network and vice versa, that is, an heuristic with lower performance on limited smallscale tests would function inadequately in large-scale scenarios. Our observation of this vulnerability is inspired by the well-known perils of extrapolation and the overfitting issue in machine learning. The point is that it should be very cautious to generalize a model from training data to unseen data without rigorous evaluation. Second, as a consequence of uncertainty in solution quality obtained from heuristic, operating a realistic network with a heuristic may result in poor network performances and worse still, may ruin the potential benefits that a new technological solution permits. Besides, the fact that comparing two designs based on non-optimal states derived by different heuristic algorithms may undermine the conclusion as the reported figure of merit can be distorted. In this work, we pinpoint those issues and provide a realistic case study to highlight and demonstrate the impact of such vulnerabilities. For the sake of clarity, the adversarial illustration provided in this paper is just centered on solving RWA and RSA problems, yet the underlying argument is broader.

\section{Optical Networks Design: Exact vs. Heuristic Solutions}

In this part, we present a typical process of designing and operating an optical network with exact and heuristic solutions. The case in point is a static planning problem where given a physical topology and a set of demands to be served, find the route and assign spectrum to each demand subject to a number of technological constraints so that all demands are served and the objective is to minimize the spectrum width. Two variants are considered, that is, RSA and RWA corresponding to elastic optical networks and fixed WDM ones. For convenience, only optimization model and heuristic for RSA are described here.

Step 1: MILP formulation for RSA problem

Given Information:

- $G(V, E)$ : Physical network topology with $|V|$ nodes and $|E|$ links. Each link $e \in E$ has its beginning node $s(e)$ and its ending node $r(e)$.

- $D$ : Set of traffic demand, indexed by $d$. Each demand $d$ has its origin $s(d)$ and destination $r(d)$ respectively and request $n_{d}$ spectrum slices 
- S: Set of spectrum slices on each link, indexed by $s$. The link capacity measured in number of spectrum slices is therefore $|S|$

$-C^{d}$ : Set of channels for demand d. Each channel $c \in C^{d}$ consists of exactly $n_{d}$ contiguous spectrum slices. Note that $\left|C^{d}\right|=|S|-n_{d}+1$

- $C=\bigcup_{d \in D} C^{d}$ : Set of all possible channels

- $w_{c, s}$ : constant equals to 1 if the channel $c$ contains spectrum slice $s, 0$ otherwise

Variables:

$-x_{e, c}^{d} \in\{0,1\}: 1$ if the link $e$ and channel $c$ is used for demand $d, 0$ otherwise.

$-\alpha_{c}^{d} \in\{0,1\}: 1$ if the channel $c$ is used for demand $d, 0$ otherwise.

$-\gamma_{e, s}: 1$ if the spectrum slice $s$ is used on link $e, 0$ otherwise

$-\delta_{s}: 1$ if the spectrum slice $s$ is used for the entire network, 0 otherwise

Objective:

Minimize $\sum_{s \in S} \delta_{s}$

Subject to the following constraints:

$\sum_{c \in C^{d}} \alpha_{c}^{d}=1 \quad \forall d \in D$

$$
\sum_{e \in E: v \equiv s(e)} x_{e, c}^{d}-\sum_{e \in E: v \equiv r(e)} x_{e, c}^{d}=
$$

$\left\{\begin{array}{ll}\alpha_{c}^{d} & \text { if } v \equiv s(d) \\ -\alpha_{c}^{d} & \text { if } v \equiv r(d) \\ 0 & \text { otherwise }\end{array} \quad \forall v \in V, \forall d \in D, \forall c \in C^{d}\right.$

$\sum_{d \in D} \sum_{c \in C^{d}} x_{e, c}^{d} \times \omega_{c, s}=\gamma_{e, s} \quad \forall e \in E, \forall s \in S$

$\sum_{e \in E} \gamma_{e, s} \leq|E| \delta_{s} \quad \forall s \in S$

The objective in Eq. 1 aims at minimizing the spectrum usage measured in terms of number of spectrum slots to support all traffic demands. Constraints in Eq. 2 guarantees that all demands are served by finding the right spectrum channel. The traditional flow conservation constraint of each demand is ensured in Eq, The spectrum slice uniqueness is enforced by Eq. 4. Finally, the definition of using a spectrum slice in the network, that is, a spectrum slice is considered to be used if it is occupied in any link of the network, is indicated by Eq. 5.

Step 2: It has been widely proven that RSA problem is NP-complete [19, 27, 33] and therefore optimization solvers are not guaranteed to finish in polynomial time. A scalable heuristic solution has to be developed to operate for realistic networks.
Step 3: As a reference heuristic algorithm for RSA problem, we choose MSF (i.e., Most Slices First), a typical reference algorithm for benchmarking purpose. Details of the algorithm could be found in [27, 36. MSF is a sequential heuristic algorithm for RSA where demands are ordered according to their number of requested spectrum slices and demands with highest number of slices is served first. The serving of each demand under the spectrum continuity, spectrum contiguity and non-overlapping condition is performed as follows.

We represent the spectrum availability in each link $e \in E$ by a vector $u_{e}$ with the size equaling to the number of spectrum slices $S$. Each element in the vector encodes the availability of the spectrum slice, that is, element $i$ equals to 1 if the slice $i$ is available and 0 if it is occupied.

$\overline{u_{e}}=\left(u_{e 1}, u_{e 2}, \ldots, u_{e|S|}\right)$

For a path $p$, we can calculate the spectrum availability vector for that path by performing AND operation for all the spectrum availability vectors of links constituting the path.

$\overline{U_{p}}=\cap_{e \in p} \overline{u_{e}}$

For each demand $d$ requesting $n_{d}$ slices, we first evaluate the spectrum availability vectors of 3 candidate paths (i.e., 3 shortest paths) and then search the first possible placement of $n_{d}$ contiguous slices. The path with the lowest indexed starting slice is selected. Afterward, we update the spectrum availability of the links constituting the selected path. After serving all demands, we evaluate this solution with a fitness function as $F=\max f\left(\overline{u_{e}}\right)$ where $f($.$) is the function that$ returns the index of the last used slice on spectrum availability vector $\overline{u_{e}}$. The value of $F$ is the objective value of that solution.

\section{Mind the Perils of Extrapolation and Performance Comparison: A Case Study}

In this part, we present a numerical case study on solving the routing and spectrum assignment (RSA), and routing and wavelength assignment (RWA) with both exact and heuristic approaches. As far as this part is concerned, that the RSA problem is solved represents the choice of elastic optical networks technologies to support a given set of traffic demands while the solving of RWA problem refers to the adoption of fixed WDM technologies. For solving the MILP models, we use CPLEX and run until the optimal solution is reached. 
Advanced implementation techniques have been performed including warm-start and parallel computing to expedite the running time.

When it comes to heuristically solving the RWA problem, the metric for evaluation is the number of used wavelengths to accommodate the same set of traffics as with the RSA problem. Here we use a genetic algorithm for finding a good order of demands to be served and then the first-fit wavelength assignment is applied. Details of the algorithm could be found in [3,40].

\subsection{Step 4: The Possible Trap of Extrapolation}

We first draw a comparison on solution quality between exact approach based on solving optimally optimization model and approximation approach based on heuristic algorithms. The conventional way to do so is first to compare on small-scale instances and if the heuristic's quality is good enough, a conclusion could be made that it is ready to apply the heuristic for large-scale models. But this is where the pitfall may occur.

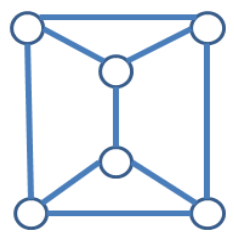

(a) Small topology (6 nodes, 18 links)

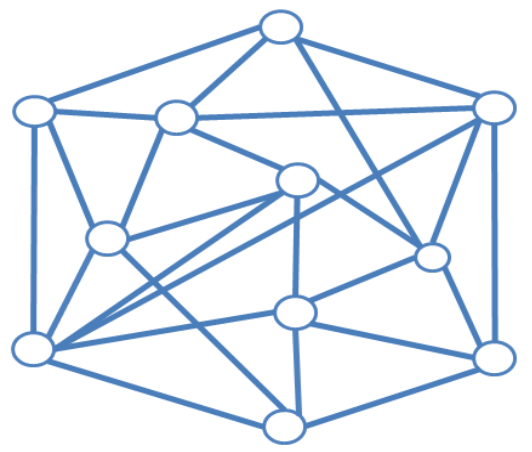

(b) COST239 (11 nodes, 52 links)

Fig. 1: Network Topologies under Test

In our numerical illustration, for small tests, we use a 6-node topology (Figure 1(a)) with 10 traffic matrices and each traffic matrix consists of 8 traffic demands whose number of requested spectrum slices is a random integer number between 1 and 4 . This corresponds to a random demand between 25 Gbps and 100 Gbps with a step of 25 Gbps operating in an flex-grid optical network adopting QPSK modulation and spectrum slice width of $12.5 \mathrm{GHz}$. Table 1 reveals the comparison of heuristic and optimal solutions for both RSA and RWA problem on small instances. Note that the number of used spectrum slices is a metric for RSA problem and each slice is $12.5 \mathrm{GHz}$ width while the number of used wavelength is a metric for RWA problem and each wavelength is $50 \mathrm{GHz}$ width. As can been seen from Table1 both RSA heuristic and RWA heuristic achieve remarkable performance as its solution is optimal in majority of instances. Indeed, only at traffic instance 5, the RSA heuristic is one slice far from its optimality. Based on that observation, the conventional declaration of some research works would be that the proposed heuristic is efficient and it is therefore ready to bring that heuristic to realistic scenarios.

Table 1: Performance Comparison between Exact Solutions and Heuristic Ones

\begin{tabular}{|c|c|c|c|c|}
\hline $\begin{array}{c}\text { Traffic } \\
\text { Instance }\end{array}$ & $\begin{array}{c}\text { Optimal } \\
\text { RSA }\end{array}$ & $\begin{array}{c}\text { Heuristic } \\
\text { RSA }\end{array}$ & $\begin{array}{c}\text { Optimal } \\
\text { RWA }\end{array}$ & $\begin{array}{c}\text { Heuristic } \\
\text { RWA }\end{array}$ \\
\hline \hline 1 & 4 & 4 & 2 & 2 \\
2 & 4 & 4 & 1 & 1 \\
3 & 4 & 4 & 1 & 1 \\
4 & 4 & 4 & 1 & 1 \\
5 & 4 & 5 & 2 & 2 \\
6 & 4 & 4 & 1 & 1 \\
7 & 4 & 4 & 1 & 1 \\
8 & 4 & 4 & 2 & 2 \\
9 & 4 & 4 & 1 & 1 \\
10 & 3 & 3 & 2 & 2 \\
\hline
\end{tabular}

For realistic network, a COST239 topology (Figure 1(b)) is employed with 10 traffic sets whose each one is made up of 45 random demands. Each demand again requests a random number of requested spectrum slices between 1 and 4 . Note that for fixed WDM network with QPSK modulation and wavelength granularity of $50 \mathrm{GHz}$, either the demand is $25 \mathrm{Gbps}$ or $100 \mathrm{Gbps}$, it is all filled with one wavelength of $50 \mathrm{GHz}$.

Let us observe the results of applying such heuristic on a realistic COST 239 topology. Different from traditional research works, we provide here the optimal solutions obtained from solving the MILP formulation to double-check the heuristic solutions. Figure 2 depicts the comparison of optimal RSA and heuristic one at different traffic instances. It can be seen that its heuristic solution deviate considerably from optimal values. Only 1 out of 10 cases, the heuristic solution reaches the optimal value while in the worst case, the gap can be up to $60 \%$. On average, the heuristic solution has a 
quality gap of $34 \%$ compared to optimal one. In other words, there has been a failure in the generalization of RSA heuristic from small cases to large ones. The consequence of that poor generalization is that if such heuristic is decided to use for designing and operating a real network, a substantial spectrum resources could be squandered due to the poor provisioning of algorithm. On the other hand, as shown in Figure 3, the RWA heuristic has an exceptional generalization capability as it can achieve optimality in 9 out of 10 cases and the average gap is therefore as low as $5 \%$.

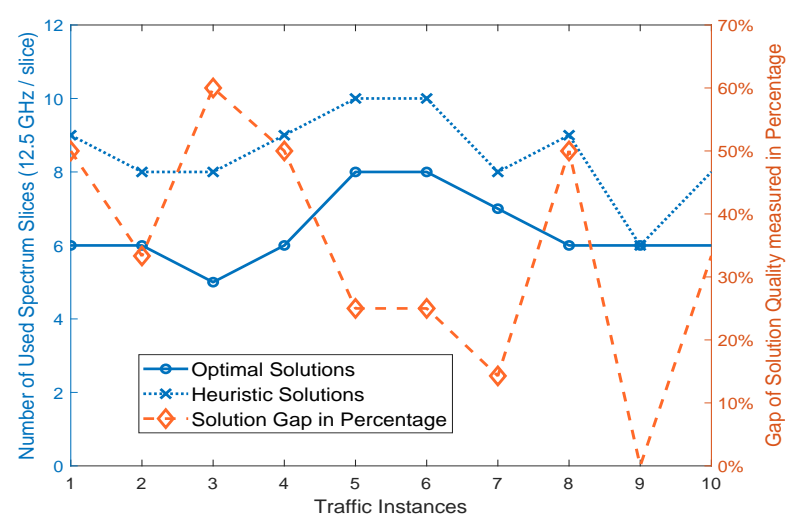

Fig. 2: Comparison between Optimal and Heuristic Solutions of RWA problem on COST239 network

Apparently both RSA and RWA heuristic are good enough under small-scale tests and yet, they behave differently upon generalization. For these particular RSA and RWA heuristic and on the particular COST239 network, the discrepancy between optimal and heuristic ones are likely due to the flexibilities of heuristic algorithms in adapting to changing network topologies. Putting the spectrum/wavelength assignment aside as it is both relied on first-fit scheme, the RSA heuristic is based on a fixed routing mechanism of finding 3 candidate paths for each demand. On a small topology of 6 -node as in Fig. 1(a), such 3 candidate paths are adequate for reaching (nearly) optimal solutions. However, when topology is changed to a more densely connected structure, such fixed 3 candidate paths routing appears to miss a large part of search space. As a result, the RSA heuristic solutions are likely deviated from the optimal ones. On the other hand, the RWA heuristic is a more advanced one permitting certain adaptation to both traffic and topology. Specifically, the Genetic Algorithm is used to find the good ordering of traffic demands for serving and for the routing, a maximum of 10 different candidate paths are allowed for each de- mand. Consequently, in some way, the RWA heuristic is more adaptive to a wide range of traffic and network topologies and therefore, justifies for its generalization capability.

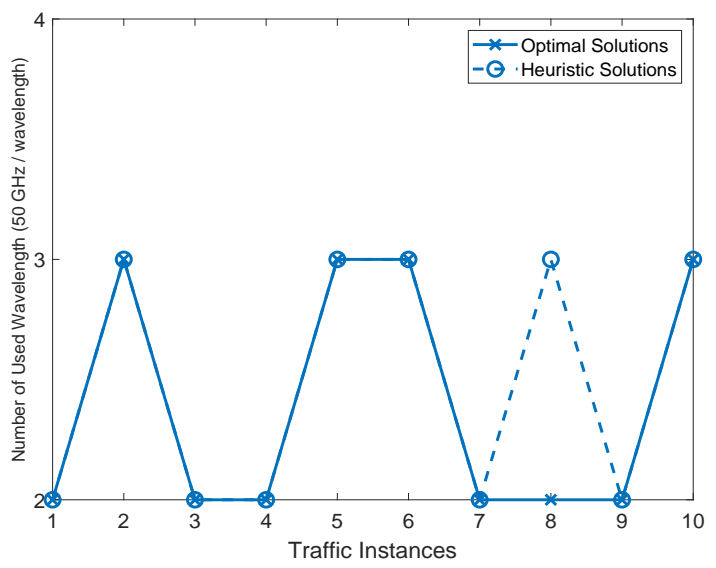

Fig. 3: Comparison between Optimal and Heuristic Solutions of RSA problem on COST239 network

Note that we have intentionally made use of such two carefully crafted heuristic to pinpoint a loophole, possibly resulting in Achilles heel when it comes to designing and operating optical networks with heuristic algorithms. The critical point to raise is that current practices of designing and testing heuristic algorithms have been performed without rigorous framework. In fact, it remains quite arbitrary for the majorities of works on the selection of small cases for heuristic algorithm testing and it has been lacking of useful metrics for measuring and/or predicting the effectiveness of heuristic algorithms with respect to specific features of network topologies and traffic demands.

\subsection{Step 5: The Possible Trap of Performance Comparison}

We now focus on the issue of performance comparison when it comes to accommodating a set of traffic demands with elastic optical networks technologies and a reference one based on WDM technologies. Basically, it is about solving the aforementioned RSA and RWA problems and comparing their solutions. The performance metric for comparison is the bandwidth which is measured by the number of used spectrum slices $\times 12.5$ $\mathrm{GHz}$ for RSA cases and the number of used wavelength $\times 50 \mathrm{GHz}$ for RWA cases. 
Although it is intuitive to predict that the elastic optical networks operating at a finer granular of spectrum will be spectrally more efficient than its counterpart fixed WDM networks, realizing that potential gain is a different story. The numerical results presented in this part illustrates that critical point.

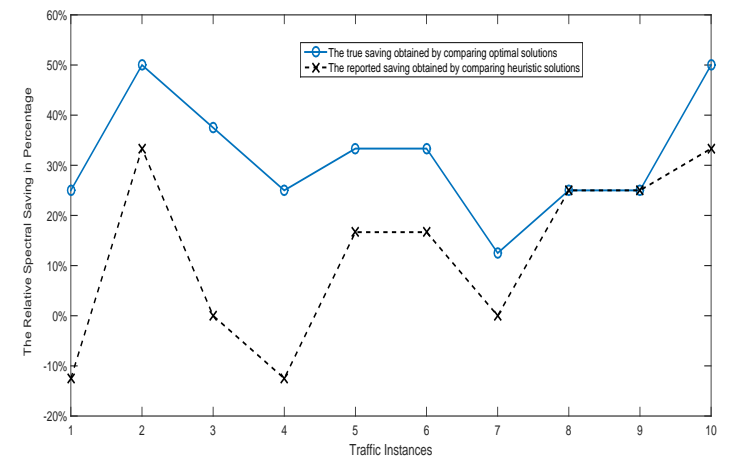

Fig. 4: Relative Spectral Saving of Elastic Optical Networks compared to WDM networks

Figure 4 showcases the relative spectral savings when RSA (i.e., elastic optical networks is employed) is solved in comparison with solving the RWA problem (i.e., fixed WDM network is employed). Very often in some research works, only the relative spectral savings obtained from comparing RSA heuristic and RWA heuristic is reported. In an attempt to detect the vulnerability of such traditional process, we include here the (true) relative spectral savings obtained from comparing the optimal solutions of both RSA and RWA problems. This is to highlight the point that how performing with heuristic might produce distorted figure of merits.

As shown in Figure 4 there are two instances (i.e., traffic index 1,4$)$ that the relative spectral saving is negative and it means that operating an elastic optical networks with the proposed RSA heuristic results in lower spectral efficiency than operating a fixed WDM network with the RWA heuristic. This counter-intuitive result is due to the low-quality of RSA heuristic when applying to the realistic COST239 network while the RWA heuristic performs adequately well. Such comparison thus results in distorted conclusions as the relative solution quality of two heuristics are not taken into account. Furthermore, the result highlights the fact that a poorly designed heuristic algorithm may ruin all the potential benefits that a new technology can bring. Indeed, as it can be seen, operating with MSF heuristic for RSA problem may cause higher usage of spec- trum resources compared to RWA counterpart in certain cases. Moreover, it is observed that how deviated the heuristic-based comparison is in reference to the true one. In this illustrative case, the reported gain concluded from relying on merely heuristic is on average roughly $20 \%$ lower than the true one based on comparison of optimal solutions.

The aforementioned numerical illustration pinpoints to a critical vulnerability that a conclusion drawn from performance comparison of two designs based on solely heuristic algorithms may misconceive the reality. This is due to the uncertainty in quality of heuristic solutions when extending to large-scale scenarios.

\section{Summary}

The peril of extrapolation and overfitting has found its way to some proposals for optical network designs and performance comparisons and it has been addressed for the first time in this paper. The first argument was that the traditional process of testing a heuristic algorithm on small-scale cases and then extend it to large-scale ones may leave a critical vulnerability as there is always a possibility that the heuristic does not generalize well for the large-scale ones. Consequently, if that ill-generalized heuristic is used for designing and operating a realistic optical network, a substantial spectrum resources could be squandered or alternatively, the potential of a new technological and architectural proposal could not be realized. The second argument was to pinpoint a loophole in comparing two designs based on merely heuristic solutions. Due to the nature of uncertainty in heuristic solution quality, conclusions drawn from comparing performances of two designs based merely on different heuristics may be unreliable in the sense that there might be either a large gap compared to the ideal one or worse still, a false outcome (i.e., positive gain to negative one or vice versa). Numerical case studies were provided to support our arguments.

As optical network designs lie at the intersection of fiber-optic communications, operation research and computer science, more concerted effort should therefore be undertaken to enable a more rigorous evaluation and more interpretable (explainable) results to network operators. It should be pointed out that network design algorithms play a key role in achieving operational efficiency of a network and thus, more robust and carefully designed algorithms should be developed. As a quick suggestion from this paper, a reasonable practice should 
be to provide (lower) bounds in addition to heuristic results to have a more informed perspective.

Albeit still primitive, this work calls for a reconsideration in designing and evaluation of algorithms for optical network design and a better way for conducting performance comparison. Perhaps, wisdoms from machine learning realm when it comes to training/testing and combining models in the form of ensemble learning to reduce the risk of overfitting could provide some hints. Further works should be carried out to identify a framework in addressing, for example, how many smallscale cases and how diversity regarding network topologies and traffic generation are needed to test before generalization. A forward-looking perspective would also be on conceiving data-driven heuristics (e.g., topology, traffic and/or bandwidth-aware) rather than currently adopted ones with limited flexibilities.

\section{Conflict of interest}

The authors declare that they have no conflict of interest.

\section{References}

1. Sabella, R., Iovanna, P., Bottari, G., Cavaliere, F.: Optical transport for industry 4.0. J. Opt. Commun. Netw. 12(8), 264-276 (2020). DOI 10.1364/JOCN.390701. URL http://jocn.osa.org/abstract.cfm?URI=jocn-12-8-264

2. Winzer, P.J., Neilson, D.T., Chraplyvy, A.R.: Fiberoptic transmission and networking: the previous 20 and the next 20 years. Opt. Express 26(18), 24,190-24,239 (2018). DOI 10.1364/OE.26.024190. URL http://www.opticsexpress.org/abstract. cfm?URI=oe-2

3. Simmons, J.M.: Optical Network Design and Planning, 2 edn. Springer Publishing Company, Incorporated (2014)

4. Dao, H., Morvan, M., Gravey, P.: An efficient networkside path protection scheme in ofdm-based elastic optical networks. International Journal of Communication Systems 31(1), e3410. DOI 10.1002/dac.3410. URL https://onlinelibrary.wiley.com/doi/abs/10.1002/dac.34t0 E3410 dac.3410

5. Hai, D.T.: Leveraging the survivable all-optical wdm network design with network coding assignment. IEEE Communications Letters 21(10), 2190-2193 (2017). DOI 10.1109/LCOMM.2017.2720661

6. Thanh, H.D., Morvan, M., Gravey, P., Cugini, F., Cerutti, I.: On the spectrum-efficiency of transparent optical transport network design with variable-rate forward error correction codes. In: 16th International Conference on Advanced Communication Technology, pp. 1173-1177 (2014). DOI 10.1109/ICACT.2014.6779143

7. Hai, D.T., Chau, L.H., Hung, N.T.: A priority-based multiobjective design for routing, spectrum, and network coding assignment problem in network-coding-enabled elastic optical networks. IEEE Systems Journal 14(2), 2358-2369 (2020). DOI 10.1109/JSYST.2019.2938590
8. Dao, T.H.: On optimal designs of transparent wdm networks with $1+1$ protection leveraged by all-optical xor network coding schemes. Optical Fiber Technology 40, 93 - 100 (2018). DOI https://doi.org/10.1016/j.yofte.2017.11.009. URL http://www.sciencedirect.com/science/article/pii/S106852001730

9. Hai, D.T., Minh, H.T., Chau, L.H.: Qos-aware protection in elastic optical networks with distanceadaptive and reconfigurable modulation formats. Optical Fiber Technology 61, 102,364 (2021). DOI https://doi.org/10.1016/j.yofte.2020.102364. URL https://www.sciencedirect.com/science/article/pii/S10685200203

10. DAO THANH, H.: Contribution to Flexible Optical Network Design: Spectrum Assignment and Protection. Theses, Télécom Bretagne ; Université de Bretagne Occidentale (2014). URL https://hal.archives-ouvertes.fr/tel-01206788

11. Hai, D.T.: A bi-objective integer linear programming model for the routing and network coding assignment problem in wdm optical networks with dedicated protection. Computer Communications 133, $51 \quad-58$ (2019). DOI https://doi.org/10.1016/j.comcom.2018.08.006. URL http://www.sciencedirect.com/science/article/pii/S0140366418300

12. Hai, D.T.: On routing, spectrum and network coding assignment problem for transparent flexgrid optical networks with dedicated protection. Computer Communications (2019). DOI https://doi.org/10.1016/j.comcom.2019.08.005. URL http://www.sciencedirect.com/science/article/pii/S0140366418306

13. Hai, D.T.: Network coding for improving throughput in wdm optical networks with dedicated protection. Optical and Quantum Electronics 51(387) (2019). DOI $10.1007 / \mathrm{s} 11082-019-2104-5$. URL https://doi.org/10.1007/s11082-019-2104-5

14. Hai, D.T.: On the spectrum-efficiency of qos-aware protection in elastic optical networks. Optik 202, 163,563 (2020). DOI https://doi.org/10.1016/j.ijleo.2019.163563. URL https://www.sciencedirect.com/science/article/pii/S00304026193

15. Hai, D.T.: An optimal design framework for $1+1$ routing and network coding assignment problem in wdm optical networks. IEEE Access 5, 22,291-22,298 (2017). DOI 18-24.19.9109/ACCESS.2017.2761809

16. Dao Thanh, H., Morvan, M., Gravey, P.: On the usage of flexible transponder in survivable transparent flexgrid optical network. In: 2014 9th International Symposium on Communication Systems, Networks Digital Sign (CSNDSP), pp. 1123-1127 (2014). DOI 10.1109/ CSNDSP.2014.6923998

Hai, D.T.: A novel adaptive operation of multi-line rate transponder for dedicated protection in wdm network. In: 2017 Seventh International Conference on Information Science and Technology (ICIST), pp. 69-74 (2017). DOI 10.1109/ICIST.2017.7926494

18. Hai, D.T., Hoang, K.M.: On the efficient use of multiline rate transponder for shared protection in wdm network. In: 2017 International Conference on Recent Advances in Signal Processing, Telecommunications Computing (SigTelCom), pp. 181-186 (2017). DOI 10.1109/ SIGTELCOM.2017.7849819

19. Varvarigos, E., Christodoulopoulos, K.: Algorithmic aspects of optical network design. In: Optical Network Design and Modeling (ONDM), 2011 15th International Conference on, pp. 1-6 (2011)

20. Varvarigos, E.A., Christodoulopoulos, K.: Algorithmic aspects in planning fixed and flexible optical networks 
with emphasis on linear optimization and heuristic techniques. Journal of Lightwave Technology 32(4), 681-693 (2014). DOI 10.1109/JLT.2013.2290842

21. Hai, D.T.: On solving the $1+1$ routing, wavelength and network coding assignment problem with a bi-objective integer linear programming model. Telecommunication Systems 71(2), 155165 (2019). DOI 10.1007/s11235-018-0474-9. URL https://doi.org/10.1007/s11235-018-0474-9

22. Nguyen, D.M., Ngoc, L.A., Huong, P.T.V., Son, N.H., Hai, D.T.: An efficient column generation approach for solving the routing and spectrum assignment problem in elastic optical networks. In: 2019 6th NAFOSTED Conference on Information and Computer Science (NICS), pp. 130-135 (2019). DOI 10.1109/NICS48868. 2019.9023831

23. Hai, D.T.: A novel integer linear programming formulation for designing transparent wdm optical core networks. In: 2019 International Conference on Advanced Technologies for Communications (ATC), pp. 273-277 (2019). DOI 10.1109/ATC.2019.8924515

24. Hai, D.T.: Re-designing dedicated protection in transparent wdm optical networks with xor network coding. In: 2018 Advances in Wireless and Optical Communications (RTUWO), pp. 118-123 (2018). DOI 10.1109/RTUWO. 2018.8587873

25. Musa, M., Elgorashi, T., Elmirghani, J.: Energy efficient survivable ip-over-wdm networks with network coding. J. Opt. Commun. Netw. 9(3), 207217 (2017). DOI 10.1364/JOCN.9.000207. URL http://jocn.osa.org/abstract.cfm?URI=jocn-9-3-207

26. Shen, G., Tucker, R.S.: Energy-minimized design for ip over wdm networks. IEEE/OSA Journal of Optical Communications and Networking 1(1), 176-186 (2009). DOI 10.1364/JOCN.1.000176

27. Christodoulopoulos, K., Tomkos, I., Varvarigos, E.A.: Elastic bandwidth allocation in flexible ofdm-based optical networks. Journal of Lightwave Technology 29(9), 1354-1366 (2011). DOI 10.1109/JLT.2011.2125777

28. Klinkowski, M., Walkowiak, K., Jaworski, M.: Off-line algorithms for routing, modulation level, and spectrum assignment in elastic optical networks. In: 2011 13th International Conference on Transparent Optical Networks, pp. 1-6 (2011). DOI 10.1109/ICTON.2011.5971109

29. Zhang, J., Zhao, Y., Yu, X., Zhang, J., Song, M., Ji, Y., Mukherjee, B.: Energy-efficient traffic grooming in sliceable-transponder-equipped ip-over-elastic optical networks [invited]. IEEE/OSA Journal of Optical Communications and Networking 7(1), A142-A152 (2015). DOI 10.1364/JOCN.7.00A142

30. Manousakis, K., Christodoulopoulos, K., Kamitsas, E., Tomkos, I., Varvarigos, E.A.: Offline impairmentaware routing and wavelength assignment algorithms in translucent wdm optical networks. J. Lightwave Technol. $\mathbf{2 7}(12), \quad 1866-1877$ (2009). URL http://j1t.osa.org/abstract.cfm?URI=jlt-27-12-1866

31. Hirano, Y., He, F., Sato, T., Oki, E.: Backup network design against multiple link failures to avoid link capacity overestimation. IEEE Transactions on Network and Service Management 17(2), 1254-1267 (2020). DOI 10.1109/TNSM.2019.2959072

32. Rottondi, C., Tornatore, M., Pattavina, A., Gavioli, G.: Routing, modulation level, and spectrum assignment in optical metro ring networks using elastic transceivers. J. Opt. Commun. Netw. 5(4), 305-315 (2013). DOI 10.1364/JOCN.5.000305. URL http://jocn.osa.org/abstract.cfm?URI=jocn-5-4-305
33. Velasco, L., Castro, A., Ruiz, M., Junyent, G.: Solving routing and spectrum allocation related optimization problems: From off-line to inoperation flexgrid network planning. J. Lightwave Technol. 32(16), 2780-2795 (2014). URL http://jlt.osa.org/abstract.cfm?URI $=j l t-32-16-2780$

34. Klinkowski, M., Ruiz, M., Velasco, L., Careglio, D., Lopez, V., Comellas, J.: Elastic spectrum allocation for time-varying traffic in flexgrid optical networks. IEEE Journal on Selected Areas in Communications 31(1), 2638 (2013). DOI 10.1109/JSAC.2013.130104

35. Tang, F., Shen, G., Rouskas, G.N.: Crosstalk-aware shared backup path protection in multi-core fiber elastic optical networks. Journal of Lightwave Technology 39(10), 3025-3036 (2021). DOI 10.1109/JLT.2021. 3064935

36. Hai, D.T., Morvan, M., Gravey, P.: Combining heuristic and exact approaches for solving the routing and spectrum assignment problem. IET Optoelectronics 12(2), 65-72 (2018). DOI 10.1049/iet-opt.2017.0013

37. Hai, D., Morvan, M., Gravey, P.: On the routing and spectrum assignment with multiple objectives. In: Advanced Photonics for Communications, p. JT3A.12. Optical Society of America (2014). URL http://www. osapublishing. org/abstract.cfm?URI=PS-2014-JT3A.12

38. Gravey, P., Hai, D., Morvan, M.: On the advantages of co-ofdm transponder in network-side protection. In: Advanced Photonics for Communications, p. PW1B.3. Optical Society of America (2014). DOI 10.1364/PS.2014.PW1B.3. URL http://www.osapublishing.org/abstract.cfm?URI=PS-2014-PW1B.3

39. Hai, D.T.: Multi-objective genetic algorithm for solving routing and spectrum assignment problem. In: 2017 Seventh International Conference on Information Science and Technology (ICIST), pp. 177-180 (2017). DOI 10.1109/ICIST.2017.7926753

40. Hai, D.T., Hoang, K.M.: An efficient genetic algorithm approach for solving routing and spectrum assignment problem. In: 2017 International Conference on Recent Advances in Signal Processing, Telecommunications Computing (SigTelCom), pp. 187-192 (2017). DOI 10.1109/SIGTELCOM.2017.7849820

41. Christodoulopoulos, K., Manousakis, K., Varvarigos, E.: Offline routing and wavelength assignment in transparent wdm networks. IEEE/ACM Transactions on Networking 18(5), 1557-1570 (2010). DOI 10.1109/TNET.2010. 2044585

42. Hai, D.T.: Three shades of partial protection in elastic optical networks (2021). ArXiv:2105.01046

43. He, S., Qiu, Y., Xu, J.: Invalid-resource-aware spectrum assignment for advanced-reservation traffic in elastic optical network. Sensors 20(15) (2020). DOI 10.3390/s20154190. URL https://www.mdpi.com/1424-8220/20/15/4190

44. Moghaddam, E.E., Beyranvand, H., Salehi, J.A.: Routing, spectrum and modulation level assignment, and scheduling in survivable elastic optical networks supporting multi-class traffic. Journal of Lightwave Technology 36(23), 5451-5461 (2018). DOI 10.1109/JLT.2018. 2874820 